\title{
Analysis of Noise under Regime Switching
}

\author{
Ling Bai ${ }^{1}$, Xiaoyue $\mathbf{L i}^{2}$ \\ ${ }^{1}$ College of Mathematics, Jilin University, Changchun, China \\ ${ }^{2}$ School of Mathematics and Statistics, Northeast Normal University, Changchun, China \\ E-mail: bailing@jlu.edu.cn
}

Received March 22, 2011; revised May 17, 2011; accepted May 20, 2011

\begin{abstract}
In this paper we consider a stochastic nonlinear system under regime switching. Given a system $\dot{x}(t)=f(x(t), r(t), t)$ in which $f$ satisfies so-called one-side polynomial growth condition. We introduce two Brownian noise feedbacks and stochastically perturb this system into $\mathrm{d} x(t)=(x(t), r(t), t) \mathrm{d} t$ $+\sigma(r(t))|x(t)|^{\beta} x(t) \mathrm{d} W_{1}(t)+q(r(t)) x(t) \mathrm{d} W_{2}(t)$. It can be proved that appropriate noise intensity may suppress the potentially explode in a finite time and ensure that this system is almost surely exponentially stable although the corresponding system without Brownian noise perturbation may be unstable system.
\end{abstract}

Keywords: Hybrid System, One-Side Polynomial Growth Condition, Ito^Formula, Stochastic Ultimate Boundedness

\section{Introduction}

Recently, there has been increasing attention devoted to the different effects of environmental noise. The first important fact is that noise can be used to stabilize a given unstable system or to make a system even more stable when it is already stable [1]. It is often mentioned that under the local Lipschitz and linear growth conditions, a n-dimensional nonlinear system

$$
\dot{x}(t)=f(x(t), t), t>0 ; x(0)=\xi \in R^{n}
$$

with $f(0, t)=0$ can be stabilized by the Brownian noise $[2,3]$. Then Appleby and Mao examined the stabilization of noise when $f$ satisfies the one-sided linear growth condition $[4,5]$.

Another important fact is that the noise can suppress the explosions (in a finite time) in population dynamics [6] which means that this fact guarantees the existence of global solutions. Deng et al. developed a general theory on the suppression of noise when $\mathrm{f}$ satisfies the one-sided linear growth condition [7]. Even in this article, authors show that the noise can make a given system whose solutions are bounded become a new system whose solutions will grow exponentially, namely, the noise can expresses exponential growth.

However, all of the papers mentioned above only consider the perturbation by Brownian noise. From another point of view, let us now take a further step by considering another type of environmental noise, namely, color noise, or say telegraph noise which can be illustrated as a switching between two or more regimes of environment, the regime switching and the environmental noise work together to make the system change. Recently, the research in this field we here mention [8,9]. Consider a nonlinear system described by an ordinary differential equation with Markovian switching of the form:

$$
\dot{x}(t)=f(x(t), r(t), t),
$$

here $f: R^{n} \times S \times R_{+} \rightarrow R^{n}$ is local Lipschitz continuous and obeys linear growth condition, or $\mathrm{f}$ obeys the one-side linear growth condition:

$$
\langle x, f(x, i, t)\rangle \leq K_{1}+K_{2}|x|^{2},
$$

the solution of this equation may grow exponentially with probability one. Guangda hu [10] Theorem 2.2 indicates if the noise is sufficiently large, it will suppress this potentially exponential growth at most polynomially. On the other hand, Guangda hu [11] also reveal that regime switching and the environmental white noise will express the exponential growth.

Although the one-side linear growth condition allows for a wider class of systems to be studied than the linear growth condition, many simple and important systems are still excluded because the coefficients of system does neither satisfy the linear growth condition nor the one-side linear growth condition. Fuke Wu [12] have devoted contributions to improve this work which extend the role of Brownian noise for suppression and stabiliza- 
tion to cover the wider systems than $[6,7]$. The authors [12] introduce the following so-called one-side polynomial growth condition they give the further assumption for $f$ :

Assumption A. There are some nonnegative numbers $\alpha, \kappa, \gamma$ such that

$$
\langle x, f(x, t)\rangle \leq \kappa|x|^{\alpha+2}+\gamma|x|^{2}
$$

for all $(x, t) \in R^{n} \times R_{+}$.

It is easy to see that $\alpha=0$ we obtain the classical one-side linear growth condition:

$$
\langle x, f(x, t)\rangle \leq(\kappa+\gamma)|x|^{2}
$$

which means that one-side polynomial growth condition generalize one-side linear growth condition. If $f$ satisfies the one-side polynomial growth condition, the system (1.1) may be explode in finite time. For example, consider a simple logistic equation

$$
\dot{x}(t)=x(1+x)
$$

with initial value $x(0)=1$, the expression of the solution has $x(t)=\frac{1}{-1+2 e^{-t}}$, there has only local solution for $1 \leq t \leq \log 2$.

Usually, only local Lipschitz and linear growth conditions guarantee a unique global solution, linear growth condition play an important role to guarantee the existence of global solutions of (1.1). Suppose $f$ only satisfy the one-side polynomial condition and local Lipschitz condition, author [12] introduce Brownian noise feedback to suppress the potential explosion of the deterministic system (1.1) and stabilize the given system.

However, little is as yet known about the properties of system satisfying the one-side polynomial growth condition under regime switching, it is therefore the motivation of our present paper to consider the system subjected to both white noise and color telegraph (hybrid system). More precisely, in this paper we will develop the theory presented in [12] to cope with systems where they are subjected to both white noise and colored noise. Given an unstable hybrid system described by an ordinary differential equation with Markovian switching

$$
\dot{x}(t)=f(x(t), r(t), t)
$$

in which $f$ satisfies the one-side polynomial growth condition under regime switching, we introduce two Brownian noise feedbacks and therefore discuss the following nonlinear hybrid system:

$$
\begin{aligned}
& \mathrm{d} x(t)=f(x(t), r(t), t) \mathrm{d} t \\
& +\sigma(r(t))|x(t)|^{\beta} x(t) \mathrm{d} W_{1}(t)
\end{aligned}
$$

or

$$
\begin{aligned}
\mathrm{d} x(t) & =f(x(t), r(t), t) \mathrm{d} t \\
& +\sigma(r(t))|x(t)|^{\beta} x(t) \mathrm{d} W_{1}(t) \\
& +q(r(t)) x(t) \mathrm{d} W_{2}(t)
\end{aligned}
$$

The next section we will show that appropriate $\beta$ may guarantee this system (1.3) or (1.4) exists a unique global solution although the corresponding hybrid system (1.2) may explode in a finite time.

\section{Positive and Global Solution}

Throughout this paper, unless otherwise specified, we let $\left(\Omega, \mathcal{F},\left\{\mathcal{F}_{t}\right\}_{t \geq 0}\right.$, P) be a complete probability space with a filtration $\left\{\mathcal{F}_{t}\right\}_{t \geq 0}$ satisfying the usual conditions (i.e. it is right continuous and $\mathcal{F}_{0}$ contains all P-null sets.) Let $W(t), t \geq 0$, be the standard Brownian motion defined on this probability space. We also denote by $R_{+}^{n}=$ $\left\{x \in R^{n}: x_{i}>0\right.$ for all $\left.1 \leq i \leq n\right\}$ and $\bar{R}_{+}^{n}=\left\{x \in R^{n}: x_{i} \geq 0\right.$ for all $1 \leq i \leq n\}$. Let $r(t)$ be a right-continuous Markov chain on the probability space taking values in a finite state space $S=\{1,2, \cdots, N\}$ with the generator $\Gamma=\left(\gamma_{u v}\right)_{N \times N}$ given by

$$
\begin{aligned}
& P\{r(t+\delta)=v \mid r(t)=u\} \\
& =\left\{\begin{array}{l}
\gamma_{u v} \delta+o(\delta), \quad \text { if } u \neq v, \\
1+\gamma_{u v} \delta+o(\delta), \text { if } u=v,
\end{array}\right.
\end{aligned}
$$

where $\delta>0$. Here $\gamma_{u v}$ is the transition rate from $u$ to $v$ and $\gamma_{u v} \geq 0$ if $u \neq v$ while

$$
\gamma_{u u}=-\sum_{v \neq u} \gamma_{u v} \text {. }
$$

We assume that the Markov chain $r(\cdot)$ is independent of the Brownian motion $W(\cdot)$. It is well known that almost every sample path of $r(\cdot)$ is a right continuous step function with a finite number of jumps in any finite subinterval of $\bar{R}_{+}=[0, \infty)$.

It is well known that local Lipschitz condition guarantees the solution of stochastic differential equation exists in $\left[t_{0}, \sigma_{\infty}\right)$, where $\sigma_{\infty}=\lim _{k \rightarrow \infty} \sigma_{k}$ and the stopping time $\sigma_{k}=T \wedge \inf \left\{t \in\left[t_{0}, T\right]:\left|x_{k}(t)\right| \geq k\right\}$. We need that $f$ is locally Lipschitz continuous, namely,

Assumption B. $f$ are locally Lipschitz continuous, that is, for each integer $k=1,2, \cdots$, there is a positive number $H_{k}$ such that $|f(x, i, t)-f(y, i, t)| \leq H_{k}|x-y|$ for all $i \in S, t \geq 0$ and those $x, y \in R^{n}$ with $|x| \vee|y| \leq k$.

Definition 2.1 Let $\sigma_{\infty}$ be a stopping time such that $t_{0} \leq \sigma_{\infty} \leq T$ a.s. An $R^{n}$-valued $\mathcal{F}_{t}$-adapted continuous stochastic process $\left\{x(t): t_{0} \leq t<\sigma_{\infty}\right\}$ is called a local solution of Equation (1.4) with initial value $x\left(t_{0}\right) \in R^{n}$, 
moreover, there is a nondecreasing sequence $\left\{\sigma_{k}\right\}_{k \geq 1}$ of stopping times such that $t_{0} \leq \sigma_{k} \uparrow \sigma_{\infty}$ a.s., and

$$
\begin{aligned}
x\left(t \wedge \sigma_{k}\right) & =x\left(t_{0}\right)+\int_{t_{0}}^{t \wedge \sigma_{k}} f(x(s), r(s), s) \mathrm{d} s \\
& +\int_{t_{0}}^{t \wedge \sigma_{k}} \sigma(r(s))|x(s)|^{\beta} x(s) \mathrm{d} W_{1}(s) \\
& +\int_{t_{0}}^{t \wedge \sigma_{k}} q(r(s)) x(s) \mathrm{d} W_{2}(s)
\end{aligned}
$$

holds for any $t \in\left[t_{0}, T\right)$ and $k \geq 1$ with probability 1 . If, furthermore,

$$
\lim _{t \rightarrow \sigma_{\infty}} \sup |x(t)|=\infty
$$

Whenever $\sigma_{\infty} \leq T$, then it is called a maximal local solution and $\sigma_{\infty}$ is called the explosion time.

Noting the function $g(x, i)=\sigma(i)|x|^{\beta} x$ satisfies locally Lipschitz condition for any $\beta \geq 0$. This together with Assumption B shows the existence of unique maximal local solution [see 8, p91 Theorem 3.15].

Lemma 2.2 Under Assumption B, for any initial value $x\left(t_{0}\right) \in R^{n}$ and $\beta \geq 0$, Equation (1.4) has a unique maximal local solution on $\left[t_{0}, \sigma_{\infty}\right)$, where $\sigma_{\infty}$ is the explosion time.

In order to have a unique global solution and avoid the linear growth condition, we generalize the one-side polynomial growth condition under regime switching:

Assumption C. There are some nonnegative numbers $\alpha, \kappa_{i}, \gamma_{i}$ such that

$$
\langle x, f(x, i, t)\rangle \leq \kappa_{i}|x|^{\alpha+2}+\gamma_{i}|x|^{2}
$$

For all $(x, i, t) \in R^{n} \times S \times R_{+}$.

In addition, consider a nonlinear stochastic differential equation with Markovian switching:

$$
\mathrm{d} x(t)=f(x(t), r(t), t) \mathrm{d} t+g(x(t), r(t), t) \mathrm{d} W(t)
$$

Throughout this paper, let $C^{2,1}\left(R^{n} \times \bar{R}_{+}^{n} \times S ; \bar{R}_{+}\right)$denote the family of all positive real-valued functions $\mathrm{V}(\mathrm{x}, \mathrm{t}, \mathrm{k})$ on $R^{n} \times \bar{R}_{+}^{n} \times S$ which are continuously twice differentiable in $\mathrm{x}$ and once in $\mathrm{t}$. If

$V \in C^{2,1}\left(R^{n} \times \bar{R}_{+}^{n} \times S ; \bar{R}_{+}\right)$, define an operator LV from $R^{n} \times \bar{R}_{+}^{n} \times S$ to $R$ by

$$
\begin{aligned}
L V(x, t, k)= & V_{t}(x, t, k) \\
& +V_{x}(x, t, k) f(x, k, t) \\
& +\frac{1}{2}\left[g^{T}(x, k, t) V_{x x}(x, t, k) g(x, k, t)\right] \\
& +\sum_{l=1}^{N} \gamma_{k l} V(x, t, l)
\end{aligned}
$$

where

$$
\begin{aligned}
& V_{t}(x, t, k)=\frac{\partial V(x, t, k)}{\partial t}, \\
& V_{x}(x, t, k)=\left(\frac{\partial V(x, t, k)}{\partial x_{n}}, \cdots, \frac{\partial V(x, t, k)}{\partial x_{n}}\right) \\
& V_{x x}(x, t, k)=\left(\frac{\partial V^{2}(x, t, k)}{\partial x_{i} \partial x_{j}}\right)_{n \times n} .
\end{aligned}
$$

For the convenience, the reader can refer to [8, p48-49] for the generalized Itô formula and a useful lemma, which often emerge in our later proof.

Now we first establish the theorem of the existence of the global solution to Equation (1.4).

Theorem 2.3 Under the conditions of Assumption B and Assumption C. If for any given initial data $x(0) \neq 0$, $i \in S, \sigma(i) \neq 0$ and $2 \beta>\alpha$, there exists a unique global solution $\mathrm{x}(\mathrm{t})$ to $(1.4)$ on $t \in[0, \infty)$.

Proof: Since the coefficients of (1.4) are locally Lipschitz, there is a unique local maximal solution $x(t)$ on $t \in\left[0, \tau_{e}\right)$, where $\tau_{e}$ is the explosion time. To show this solution is actually global and we need to show that $\tau_{e}=\infty$ a.s.

Let $m_{0}>0$ be sufficiently large such that every component of $x(0)$ is contained within the interval $\left(\frac{1}{m_{0}}, m_{0}\right)$. For each $m \geq m_{0}$, we define

$$
\begin{aligned}
& \tau_{m}=\inf \left\{t \in\left[0, \tau_{e}\right): x_{i}(t) \notin\left(\frac{1}{m}, m\right)\right. \\
& \text { for some } i=1,2, \cdots, n .\}
\end{aligned}
$$

Clearly $\tau_{m}$ is increasing as $m \rightarrow \infty$ and $\lim _{m \rightarrow \infty} \tau_{m}=: \tau_{\infty} \leq \tau_{e}$, if we can obtain that $\tau_{\infty}=\infty$ a.s., then $\tau_{e}=\infty$ and $x(t) \in R_{+}^{n}$ a.s. for all $t \geq 0$. That is, to complete the proof, all we need to show is that $\tau_{\infty}=\infty$ a.s. . This also equivalent to prove that, for any $\mathrm{t}>0, P\left(\tau_{m} \leq t\right) \rightarrow 0$ as $m \rightarrow \infty$. For $p \in(0,1)$, define a $\mathrm{C}^{2}$-function: $R_{+}^{n} \times S \rightarrow \bar{R}_{+}$by

$$
V(x, k)=C(k)|x(t)|^{p}
$$

If $x=\left(x_{1}, \cdots, x_{n}\right)^{T} \in R_{+}^{n}$ for $t>0$, one can apply Itô formula to compute that for any $t \in\left[t_{0}, \tau_{e}\right)$,

$$
\begin{aligned}
\mathrm{d} V(x, k) & =L V(x, k) \mathrm{d} t \\
& +p C(k) \sigma(k)|x(t)|^{\beta+p} \mathrm{~d} W_{1}(t) \\
& +p C(k) q(k)|x(t)|^{p} \mathrm{~d} W_{2}(t)
\end{aligned}
$$

where $L V$ is defined as 


$$
\begin{aligned}
L V(x, k) & =p C(k)|x|^{p-2}\langle x, f(x, k, t)\rangle \\
& +\frac{p(p-1)}{2} q^{2}(k) C(k)|x|^{p} \\
& +\frac{p(p-1)}{2} \sigma^{2}(k) C(k)|x|^{2 \beta+p} \\
& +\sum_{l=1}^{N} \gamma_{k l} V(x, l)
\end{aligned}
$$

Let $\tilde{q}=\max _{k, l \in S}\left\{\frac{C(l)}{C(k)}\right\}$. For any $k, l \in S$, we get

$$
V(x, l)=C(l)|x|^{p} \leq \tilde{q} C(k)|x|^{p}=\tilde{q} V(x, k)
$$

Therefore,

$$
\sum_{l=1}^{N} \gamma_{k l} V(x, l) \leq \tilde{q} \sum_{l=1}^{N}\left|\gamma_{k l}\right| V(x, k)
$$

According to Assumption C, combined with (2.3) we therefore have

$$
\begin{aligned}
L V(x, k) \leq & p C(k)|x|^{p-2}\left(\kappa_{k}|x|^{\alpha+2}+\gamma_{k}|x|^{2}\right) \\
& +\frac{p(p-1)}{2} C(k) q^{2}(k)|x|^{p} \\
& +\frac{p(p-1)}{2} \sigma^{2}(k) C(k)|x|^{2 \beta+p} \\
& +\sum_{l=1}^{N}\left|\gamma_{k l}\right| V(x, l) \\
& \leq \frac{p(p-1)}{2} \sigma^{2}(k) C(k|x|)^{2 \beta+p} \\
& +p C(k) \kappa_{k}|x|^{\alpha+p} \\
& +\left(p C(k) \gamma_{k}+\frac{p(p-1)}{2} C(k) q^{2}(k)\right. \\
& +\tilde{q} \sum_{l=1}^{N}\left|\gamma_{k l}\right| C(k)|x|^{p}
\end{aligned}
$$

Noting that $p \in(0,1)$, for any $i \in S, \quad \sigma(i) \neq 0$, $C(i)>0$ and $2 \beta>\alpha$, by the boundedness of polynomial functions, there is a constant $H_{k}$ such that

$$
L V(x, k) \leq H_{k} \leq \max _{k \in S} H_{k}:=H,
$$

Therefore we get

$$
\begin{aligned}
& \mathrm{EV}\left(x\left(\tau_{m} \wedge T\right), r\left(\tau_{m} \wedge T\right)\right) \\
& \leq V(x(0), r(0))+\mathrm{E} \int_{0}^{\tau_{m} \wedge T} L V(x(s), r(s)) \mathrm{d} s \\
& \leq V(x(0), r(0))+H T:=H_{T}
\end{aligned}
$$

where $H_{T}$ is independent of $\mathrm{m}$.

Let $\Omega_{m}=\left\{\tau_{m} \leq T\right\}$ for $m \geq m_{1}$, noting that for every $\varpi \in \Omega_{m}$, there is some $\mathrm{m}$ such that $x_{m}\left(\tau_{m}, \varpi\right)$ equals either $\mathrm{m}$ or $1 / \mathrm{m}$, hence

$$
\begin{aligned}
& P\left(\tau_{m} \leq T\right) \min _{i \in S}\left\{c(i) m^{p} \wedge c(i) \frac{1}{m^{p}}\right\} \\
& \leq P\left(\tau_{m} \leq T\right) V\left(x\left(\tau_{m}\right), i\right) \\
& \leq \mathrm{E}\left[I_{\left\{\tau_{m} \leq T\right\}} V\left(x\left(\tau_{m} \wedge T\right), r\left(\tau_{m} \wedge T\right)\right)\right] \\
& \leq \mathrm{EV}\left(x\left(\tau_{m} \wedge T\right), r\left(\tau_{m} \wedge T\right)\right) \leq H_{T}
\end{aligned}
$$

Letting $m \rightarrow \infty$ implies that

$$
\lim _{m \rightarrow \infty} \sup P\left(\tau_{m} \leq T\right)=0,
$$

So we must obtain $\tau_{\infty}=\infty$ a.s., as required. The proof is complete.

Remark: The key of this proof in Theorem 2.3 is the boundedness of $L V(x, k)$ under the assumption $\sigma(i) \neq 0$ which only depends on the $2 \beta>\alpha$. This implies that the Brownian noise $\sigma(r(t))|x(t)|^{\beta} x(t) d W_{1}(t)$ plays a crucial role to suppress potential explosion of the solution and guarantees the existence of the global solution. Therefore let $q(i)=0$ we still obtain the existence of global solution of Equation (1.3).

Theorem 2.4 Under the conditions of Assumption B and Assumption $C$. If for any given initial data $x(0) \neq 0, i \in S, \sigma(i) \neq 0$ and $2 \beta>\alpha$, there exists a unique global solution $x(t)$ to (1.3) on $t \in[0, \infty)$.

\section{Stochastic Ultimate Boundedness}

Theorem 2.3 shows that the solution of SDE (1.4) with a given positive initial value will not explode. This nice property provides us with a great opportunity to discuss how the solution varies in $R_{+}^{n}$ in more details. In this section, we will give the definition of asymptotically bounded in $p$ th moment and then give some sufficient conditions which guarantee SDE (1.4) is stochastically ultimate boundedness.

Definition 3.1 The solutions $x(t)$ of SDE (1.4) are said to be asymptotically bounded in $p$ th moment if there is a positive constant $H$ such that the solution of SDE (1.4) with a given initial value has the property that

$$
\limsup _{t \rightarrow \infty} \mathrm{E}|x(t)|^{p} \leq H .
$$

For all $(t, x, i) \in R_{+} \times R^{n} \times S$.

Definition 3.2 SDE (1.4) is said to be stochastically ultimate boundedness if for any $\varepsilon \in(0,1)$, there exist positive constants $\chi=\chi(\varepsilon)$ such that

$$
\lim _{t \rightarrow+\infty} \sup P\{|x(t)| \leq \chi\} \geq 1-\varepsilon
$$

where $x(t)$ is the solution of SDE (1.4) with any positive initial value. 
In the light of Markov inequality, it is obvious that if a stochastic equation is $p$-th moment boundedness, its solutions must be stochastically ultimately bounded. So we will begin with the following lemma and make use of it to obtain the stochastically ultimate boundedness of SDE (1.4).

Lemma 3.3 Under the conditions of Assumption B and $\mathrm{C}$, for any $p \in(0,1)$, if for any $i \in S, \sigma(i) \neq 0$ and $2 \beta>\alpha$, there exists a constant $K_{p}$ such that the global solution $x(t)$ of SDE (1.4) with any given positive initial value has the property that

$$
\limsup _{t \rightarrow \infty} \mathrm{E}\left(|x(t)|^{p}\right) \leq K_{p}
$$

where $K_{p}$ is dependent on $p$ and independent of the initial value.

Proof: First, Theorem 2.3 indicates that the solution $\mathrm{x}(\mathrm{t})$ of (1.4) will remain in $R_{+}^{n}$ for all $t \geq 0$ with probability 1. For any $\varepsilon>0$ and $p \in(0,1)$, applying the Itô formula to $e^{\varepsilon t} V(x, k)$ and taking expectation yields:

$$
\begin{aligned}
& \mathrm{EV}(x, k)=e^{-\varepsilon t} V(x(0), r(0)) \\
& +e^{-\varepsilon t} E \int_{0}^{t} e^{\varepsilon s}[L V(x(s), k)+\varepsilon V(x(s), k)] \mathrm{d} s .
\end{aligned}
$$

Here $L V(x, k)$ is defined as (2.2). Therefore, by the Assumption $\mathrm{C}$ and (2.4), we have

$$
\begin{aligned}
& L V(x, k)+\varepsilon V(x, k) \\
& \leq \frac{p(p-1)}{2} C(k) \sigma^{2}(k)|x|^{2 \beta+p} \\
& +p C(k) \kappa_{k}|x|^{\alpha+p} \\
& +\left(p C(k) \gamma_{k}+\frac{p(p-1)}{2} C(k) q^{2}(k)\right. \\
& \left.+\tilde{q} \sum_{l=1}^{N}\left|\gamma_{k l}\right| C(k)+\varepsilon C(k)\right)|x|^{p}
\end{aligned}
$$

Notice that if $p \in(0,1)$ and $2 \beta>\alpha,(3.3)$ has upper boundedness which means to

$$
\begin{aligned}
& L V(x, k)+\varepsilon V(x, k) \leq \psi_{p}(k) \\
& \leq \max _{k \in S}\left\{\psi_{p}(k)\right\}=: \tilde{\psi}_{p}
\end{aligned}
$$

which implies that

$$
\begin{aligned}
& E V(x, k)=e^{-\varepsilon t} V(x(0), r(0)) \\
& +e^{-\varepsilon t} E \int_{0}^{t} e^{\varepsilon s} \tilde{\psi}_{p} \mathrm{~d} s,
\end{aligned}
$$

Namely,

$$
E V(x, k)=e^{-\varepsilon t} V(x(0), r(0))+\frac{\tilde{\psi}_{p}}{\varepsilon}\left(1-e^{-\varepsilon t}\right)
$$

Clearly,

$$
\limsup _{t \rightarrow \infty} \operatorname{EV}(x, k) \leq \frac{\tilde{\psi}_{p}}{\varepsilon}
$$

Noting the expression of $V(x, k)=C(k)|x(t)|^{p}$ denote $\hat{C}=\min _{k \in S} C(k)$, which gives

$$
\limsup _{t \rightarrow \infty} \mathrm{E}|x|^{p} \leq \frac{\tilde{\psi}_{p}}{\hat{C} \varepsilon}=: K_{p}
$$

This means that the solution is bounded in the $p$ th moment, the stochastically ultimate boundedness will follow directly. It shows the solution trajectory is bounded with large probability.

Theorem 3.4 The solution of Equation (1.4) is stochastically ultimately boundedness under the condition Lemma 3.3 , that is for any $\varepsilon \in(0,1)$, there is a positive constant $\chi(=\chi(\varepsilon))$ such that for any positive initial value, the solution of (1.4) has the property that

$$
\lim _{t \rightarrow+\infty} \sup P\{|x(t)|>\chi\}<\varepsilon \text {. }
$$

Proof: This can be easily verified by Chebyshev's inequality and Lemma 3.3 by choosing $\chi=\left(\frac{K_{p}}{\varepsilon}\right)^{\frac{1}{p}}$ sufficiently large because of the following

$$
\lim _{t \rightarrow+\infty} \sup P\{|x(t)| \leq \chi\} \geq 1-\frac{\lim _{t \rightarrow+\infty} \sup E\left[|x|^{p}\right]}{\chi^{p}} \geq 1-\varepsilon
$$

as required.

Clearly, these boundedness results are also only dependent on the choice of $\beta$ under the condition $\sigma(i) \neq 0$ and independent of $q$, so there are similar boundedness results for the Equation (1.3).

Theorem 3.5 The solution of Equation (1.3) is stochasitically ultimately bounded under the condition Lemma 3.3 , that is for any $\varepsilon \in(0,1)$, there is a positive constant $\chi(=\chi(\varepsilon))$ such that for any positive initial value, the solution of (1.3) has the property (3.5).

\section{Stabilization of Noise}

From Section 2 and 3, we know the Brownian noise $\sigma(i)|x(t)|^{\beta}|x(t)| \mathrm{d} W_{1}(t)$ can suppress the potential explosion of the solution and guarantee this global solution to be bounded in the sense of the $p$ th moment. This section is devoted to consider the effect of noise $q(i) x(t) \mathrm{d} W_{2}(t)$, we will show that some sufficiently large $q(i)$ may stabilize the system (1.4).

Especially, the hybrid system always switch from any regime to another regime, so it is reasonable to assume that the Markov chain $r(t)$ is irreducible, which equivalent to the condition that irreducible Markov chain has a 
unique stationary probability distribution $\pi=\left(\pi_{1}, \pi_{2}, \cdots, \pi_{N}\right) \in R^{1 \times N}$ which can be determined by solving the following linear equation $\pi \Gamma=0$ subject to $\sum_{k=1}^{N} \pi_{k}=1$ and $\pi_{k}>0$ for any $k \in S$, where $\Gamma$ is generator $\Gamma=\left(\gamma_{u v}\right)_{N \times N}$.

Theorem 4.1 Suppose the Markov chain $r(t)$ is irreducible, under Assumption $\mathrm{B}$ and $\mathrm{C}$, if for $\delta \in(0,1)$, $k \in S, \sigma(k) \neq 0$ and $2 \beta>\alpha$, the solution $x(t)$ of $\operatorname{SDE}(1.4)$ with any positive initial value has the property

$$
\limsup _{t \rightarrow \infty} \frac{1}{t} \log |x(t)| \leq \sum_{j=1}^{N} \pi_{j}\left[\phi_{j}-\frac{q_{j}^{2}}{2}\right] \text { a.s. }
$$

where

$$
\phi_{k}=\max _{x>0}\left\{-\frac{\sigma^{2}(k)(1-\delta)}{2}|x(s)|^{2 \beta}+\kappa(k)|x(s)|^{\alpha}+\gamma(k)\right\} .
$$

In particular, the nonlinear hybrid system (1.4) is almost surely exponentially stable if

$$
\sum_{j=1}^{N} \pi_{j}\left[\phi_{j}-\frac{q_{j}^{2}}{2}\right]<0 .
$$

Proof: By Theorem 2.3, the solution $x(t)$ with positive initial value will remain in $R_{+}^{n}$ for all $t \geq 0$. Applying the Itô formula to the function $\log |x(t)|$ leads to

$$
\begin{aligned}
& \log |x(t)|=\log |x(0)| \\
& +\int_{0}^{t}|x(t)|^{-2}\langle x(s), f(x(s), r(s), s)\rangle \mathrm{d} s \\
& -\int_{0}^{t} \frac{1}{2}\left[\sigma(r(s))^{2}|x(s)|^{2 \beta}+q^{2}(r(s))\right] \mathrm{d} s \\
& +\int_{0}^{t} \sigma(r(s))|x(s)|^{\beta} \mathrm{d} W_{1}(s)+q(r(s)) \mathrm{d} W_{2}(s)
\end{aligned}
$$

Define

$$
M(t)=\int_{0}^{t} \sigma(r(s))|x(s)|^{\beta} \mathrm{d} W_{1}(s),
$$

Clearly $M(t)$ is a continuous local martingale with the quadratic variation

$$
\langle M(t), M(t)\rangle=\int_{0}^{t} \sigma^{2}(r(s))|x(s)|^{2 \beta} \mathrm{d} s .
$$

For any $\delta \in(0,1)$, choose $\vartheta>0$ such that $\delta \vartheta>1$ and each positive integer $n>0$, the exponential martingale inequality yields

$$
\begin{aligned}
& \mathrm{P}\left\{\sup _{0 \leq t \leq n}\left[M(t)-\frac{\delta}{2} \int_{0}^{t} \sigma^{2}(r(s))|x(s)|^{2 \beta} \mathrm{d} s\right] \geq \log n^{\vartheta}\right\} \\
& \leq \frac{1}{n^{\delta \vartheta}}
\end{aligned}
$$

Since $\sum_{n=1}^{\infty} n^{-\delta \vartheta}<\infty$, by the Borel-Cantelli lemma, there exists an $\Omega_{0} \subset \Omega$ with $\mathrm{P}\left(\Omega_{0}\right)=1$ such that for any $\varpi \in \Omega_{0}$, there exists an integer $n(\varpi)$, where $n>n(\varpi)$ and $n-1 \leq t \leq n$,

$$
M(t) \leq \frac{\delta}{2} \int_{0}^{t} \sigma^{2}(r(s))|x(s)|^{2 \beta} \mathrm{d} s+\vartheta \delta \log (t+1) .
$$

This, together with Assumption C, noting the definition of (4.2), we therefore have

$$
\begin{aligned}
\log |x(t)| \leq & \log x(0)+\int_{0}^{t}\left[-\frac{\sigma^{2}(r(s))(1-\delta)}{2}|x(s)|^{2 \beta}\right. \\
& \left.+\kappa(r(s))|x(s)|^{\alpha}+\gamma(r(s))-\frac{q^{2}(r(s))}{2}\right] \mathrm{d} s \\
& +\tilde{q} W_{2}(t)+\vartheta \delta \log (t+1)
\end{aligned}
$$

where $\tilde{q}=: \max _{k \in S}\{q(k)\}$. Applying the strong law of large number [3] to the Brownian motion, we therefore have

$$
\lim _{t \rightarrow \infty} \frac{W_{2}(t)}{t}=0 \quad \text { a.s. }
$$

Moreover, by the ergodic property of the Markov chain, we have

$$
\begin{aligned}
& \lim _{t \rightarrow \infty} \frac{1}{t} \int_{0}^{t}\left[\phi(r(s))-\frac{q^{2}(r(s))}{2}\right] \mathrm{d} s \\
& =\sum_{j=1}^{N} \pi_{j}\left[\phi_{j}-\frac{q_{j}^{2}}{2}\right] \quad \text { a.s. }
\end{aligned}
$$

Combined (4.5) and (4.6), it follows from (4.4)

$$
\limsup _{t \rightarrow \infty} \frac{1}{t} \log |x(t)| \leq \sum_{j=1}^{N} \pi_{j}\left[\phi_{j}-\frac{q_{j}^{2}}{2}\right] \quad \text { a.s. }
$$

Thus the assertion (4.1) follows.

Clearly, if

$$
\sum_{j=1}^{N} \pi_{j}\left[\phi_{j}-\frac{q_{j}^{2}}{2}\right]<0,
$$

System (1.4) is almost surely exponentially stable, the proof is complete.

Remark: The condition (4.7) show the overall behavior as the result of Markovian switching, system (1.4) will be almost surely exponentially stable, but for any subsystem of (1.4), i.e.

$$
\begin{aligned}
\mathrm{d} x(t) & =f(x(t), i, t) \mathrm{d} t \\
& +\sigma(i)|x(t)|^{\beta} x(t) \mathrm{d} W_{1}(t) \\
& +q(i) x(t) \mathrm{d} W_{2}(t)
\end{aligned}
$$


may be written as

$$
\begin{gathered}
d x(t)=x(t)(1+x(t)) d t \\
+x^{2}(t) d W_{1}(t)
\end{gathered}
$$

with $x(0)=1$ when $t \geq 0$. Applied the condition (4.2) with $q(i)=0, \sigma(i)=\kappa(i)=\gamma(i)=\alpha(i)=\beta(i)=1$ to the system (4.8) yields

$$
\phi(i)=\max _{x>0}\left\{-\frac{1}{2} x^{2}+x+1\right\}=\frac{3}{2},
$$

Satisfying $\phi(i)-\frac{q^{2}(i)}{2}>0$. In [12], it shows that the trajectory of (4.8) will not tend to 0 although it has global solution.

\section{References}

[1] R. Z. Hasminskii, "Stochastic Stability of Differetial Equations," Sijthoff and Noordhoof, Alphen, 1980.

[2] X. Mao, "Exponential Stability of Stochastic Differential Equations," Dekker, New York, 1994.

[3] X. Mao, "Stochastic Differential Equations and Their Applications," Horwood Publishing, Chichester, 1997.

[4] X. Mao, "Stability and Stabilizition of Stochastic Differetnial Delay Equations," Proceeding of IET on Control and Theory and Application, November 2007, pp. 1551-1566. doi: 10.1049/iet-cta:20070006

[5] J. A. D. Appleby, X. R. Mao and A. Rodkina, "Stabilization and Destabilization of Nonlinear Differential Equations by Noise," IEEE Transactions on Automatic Control, Vol. 53, No. 3, 2008, pp. 683-691. doi:10.1109/TAC.2008.919255
[6] X. Mao, G. Marion and E. Renshaw, "Environmental Brownian Noise Suppresses Explosions in Population Dynamics," Stochastic Processes and Their Applications, Vol. 97, No. 1, 2002 pp. 95-110. doi:10.1016/S0304-4149(01)00126-0

[7] F, Q. Deng, Q. Luo; X. R. Mao and S. L. Pang, "Noise Suppresses or Expresses Exponential Growth," Systems Control Letters, Vol. 57, No. 3, 2008. doi:10.1016/j.sysconle.2007.09.002

[8] X. Mao and C. Yuan, "Stochastic Differential Equations with Markovian Switching," Imperial College Press, London, 2006.

[9] X. R. Mao; G. G. Yin and C. G. Yuan, "Stabilization and Destabilization of Hybrid Systems of Stochastic Differential Equations," Vol. 43, No. 2, 2007, pp. 264-273. doi:10.1016/j.automatica.2006.09.006

[10] G. D. Hu, M. Z. Liu, X. R. Mao and M. H. Song, "Noise Expresses Exponential Growth under Regime Switching," Systems Control Letters, Vol. 58, No. 9, 2009, pp. 691-699. doi:10.1016/j.sysconle.2009.06.006

[11] G. D. Hu, M. Z. Liu, X. R. Mao and M. H. Song, "Noise Suppresses Exponential Growth under Regime Switching," Journal of Mathematical Analysis and Applications, Vol. 355, No. 2, 2009, pp. 783-795. doi:10.1016/j.jmaa.2009.02.009

[12] F. Wu and S. G. Hu, "Suppression and Stabilization of Noise," International Journal of Control, Vol. 82, No. 11, 2009, pp. 2150-2157.

[13] C. Zhu and G. Yin, "Asymptotic Properties of Hybrid Diffusion Systems," SIAM Journal on Control and Optimazation, Vol. 46, No. 4, 2007, pp. 1155-1179. doi: $10.1137 / 060649343$ 